\title{
Study on collapsibility of typical structural loess by multi- parameter comparative analysis
}

\author{
Liu Hongcheng ${ }^{1}$, Lu Changwei ${ }^{1 *}$, Wang Yinxia ${ }^{2}$, Song Yi ${ }^{1}$, Guan Xiangfeng ${ }^{1}$, Fu Rui ${ }^{1}$, Cao Jingfeng ${ }^{1}$, Yang Zhao ${ }^{3}$ \\ ${ }^{1}$ Jilin Province Water Conservancy and Hydropower Survey and Design Institute, China \\ ${ }^{2}$ Jilin Jianyuan Design Group Co. Ltd, China \\ ${ }^{3}$ Henan Daming Architectural Design Co. Ltd, China
}

\begin{abstract}
To study the collapsibility of typical loess and its influencing factors in different areas, the samples in Jingyang, Lanzhou and Yili were studied. The correlation between initial moisture content, dry density, composition of particle size, structural parameters and the coefficient of subsidence is analyzed. The results show that: the coefficient of collapsibility is negatively correlated with the initial moisture content and dry density. In the experiments of multiple groups, the collapsibility coefficient has a certain correlation with the clay content, but not a uniform correlation with the particle content. The collapse coefficient is approximately positively correlated with the composite structure potential.
\end{abstract}

\section{Introduction}

Loess is a special soil formed in the Quaternary Period and widely distributed in the world. The total area of loess in China is about $640,000 \mathrm{~km}^{2}$, of which about $60 \%$ is collapsible loess [1]. The special material composition and structure of loess make it have special engineering properties, such as collapsibility. These special properties are easy to cause a variety of geological disasters and engineering problems, such as slope instability, foundation settlement and tunnel collapse.

Due to the influence of hydrogeological conditions and climate, the engineering properties of loess in different areas are obviously different, which increases the complexity of engineering problems in loess distribution areas. Therefore, the study of loess collapsibility and its controlling factors in different areas is of great significance to the prevention and control of geological disasters and the safety of engineering construction in loess distribution areas. Many engineering problems in loess area are directly caused by loess collapsibility deformation and its mutation. In view of these problems, some scholars have explored the calculation model of unsaturated loess collapse deformation, and studied the influence of discontinuous distribution of collapsible units on the law of collapse deformation [5][6]. However, the engineering properties of loess are the result of many factors. It is the key to study the collapsibility of loess to clarify the influencing factors and the effects of each factor. In recent years, many achievements have been made in this field. Some scholars have explored the influence of physical and mechanical properties such as water content, dry density, plastic index and pressure on loess collapsibility, and the results show that the collapsibility coefficient has a good correlation with the above parameters [7]. By comparing the collapsibility characteristics of undisturbed and compacted loess, some scholars explored the influence of structure on collapsibility from a macro perspective [13]. Some other scholars have qualitatively and quantitatively analyzed the types of loess microstructure and the influence of various experimental parameters on collapsibility from the microscopic perspective [14][15].

Most of the existing studies take loess in a certain area as the research object and explore the influence of one or several factors on collapsibility. However, the collapsibility of loess is very regional, so the loess in different areas cannot be generalized. Therefore, this study takes the loess in Jingyang, Lanzhou and Yili as the research object to analyze the effects of particle size, density, humidity and structure on loess collapsibility, in order to provide a certain theoretical basis for loess collapsibility research, engineering construction and geological disaster prevention and control in different loess areas. Therefore, this paper takes loess samples from Jingyang, Lanzhou and Yili as the research objects to analyze the effects of particle size, density, humidity and structure on loess collapsibility, in order to provide certain theoretical basis for loess collapsibility research, engineering construction, geological disaster prevention and control in different loess areas.

\footnotetext{
* Corresponding author: Lu Changwei, lcw_jsy@163.com
} 


\section{Materials and methods}

\subsection{Materials}

Soil samples were collected from Jingyang County, Shaanxi Province (E10847'51.37", N3450'32.53"), Lanzhou City, Gansu Province (E10352'37.16", N35 53'49.71") and Yili City, Xinjiang Province (E81'43'27.16", N4349'38.31"). Samples were taken in exploratory Wells ranging from $1 \mathrm{~m}$ to $19 \mathrm{~m}$ at the sampling sites. The basic physical properties of each soil sample were tested, and the results were shown in Table 1. As can be seen from the data in the table, with the increase of sampling depth, the moisture content and dry density of soil samples at the three sampling locations showed an increasing trend. In general, the dry density of the loess in Jingyang is similar to that in Lanzhou, while the dry density of the loess in Yili is slightly lower, but its plasticity index is higher, which may be caused by the higher clay content.

Table1. Basic physical properties of soil samples

\begin{tabular}{|c|c|c|c|c|c|c|c|}
\hline \multirow{2}{*}{$\begin{array}{l}\text { Location } \\
\text { of } \\
\text { samples }\end{array}$} & \multirow{2}{*}{$\begin{array}{l}\text { Number } \\
\text { of soil } \\
\text { samples }\end{array}$} & Depth & Density & $\begin{array}{c}\text { Natural moisture } \\
\text { content }\end{array}$ & $\begin{array}{c}\text { Dry } \\
\text { density }\end{array}$ & $\begin{array}{l}\text { Void } \\
\text { ratio }\end{array}$ & $\begin{array}{l}\text { Index of } \\
\text { plasticity }\end{array}$ \\
\hline & & $\mathrm{m}$ & Gs & $\omega / \%$ & $\rho_{\mathrm{d}} / \mathrm{g} \cdot \mathrm{cm}^{-3}$ & $\mathrm{e}$ & $\mathrm{I}_{\mathrm{p}}$ \\
\hline \multirow{10}{*}{ Jingyang } & JY01 & 1 & 2.71 & 11.8 & 1.32 & 1.053 & 12 \\
\hline & JY02 & 3 & & 13.2 & 1.37 & 0.978 & 13 \\
\hline & JY03 & 5 & 2.71 & 14.4 & 1.35 & 0.993 & 13 \\
\hline & JY04 & 7 & & 14.1 & 1.39 & 0.950 & 11 \\
\hline & JY05 & 9 & 2.71 & 15.8 & 1.38 & 0.964 & 14 \\
\hline & JY06 & 11 & & 16.3 & 1.42 & 0.908 & 12 \\
\hline & JY07 & 13 & 2.71 & 17.5 & 1.43 & 0.895 & 15 \\
\hline & JY08 & 15 & & 18.1 & 1.45 & 0.869 & 13 \\
\hline & JY09 & 17 & & 18.2 & 1.43 & 0.895 & 11 \\
\hline & JY10 & 19 & 2.71 & 17.8 & 1.47 & 0.844 & 12 \\
\hline \multirow{10}{*}{ Lanzhou } & LZ01 & 1 & 2.70 & 7.8 & 1.32 & 1.045 & 8 \\
\hline & LZ02 & 3 & & 8.9 & 1.34 & 1.015 & 7 \\
\hline & LZ03 & 5 & 2.70 & 9.6 & 1.35 & 1.000 & 8 \\
\hline & LZ04 & 7 & & 10.9 & 1.33 & 1.030 & 9 \\
\hline & LZ05 & 9 & 2.71 & 12.4 & 1.37 & 0.978 & 8 \\
\hline & LZ06 & 11 & & 14.7 & 1.41 & 0.915 & 10 \\
\hline & LZ07 & 13 & 2.70 & 15.3 & 1.43 & 0.888 & 7 \\
\hline & LZ08 & 15 & & 16.0 & 1.46 & 0.849 & 9 \\
\hline & LZ09 & 17 & & 16.2 & 1.45 & 0.862 & 8 \\
\hline & LZ10 & 19 & 2.71 & 15.9 & 1.48 & 0.831 & 8 \\
\hline \multirow{10}{*}{ Yili } & YL001 & 1 & 2.71 & 8.8 & 1.26 & 1.151 & 15 \\
\hline & YL02 & 3 & 2.71 & 10.6 & 1.25 & 1.168 & 14 \\
\hline & YL03 & 5 & & 12.9 & 1.28 & 1.117 & 12 \\
\hline & YL04 & 7 & 2.71 & 14.9 & 1.28 & 1.117 & 12 \\
\hline & YL05 & 9 & & 14.0 & 1.30 & 1.085 & 16 \\
\hline & YL06 & 11 & 2.70 & 16.2 & 1.31 & 1.061 & 14 \\
\hline & YL07 & 13 & & 17.7 & 1.35 & 1.007 & 13 \\
\hline & YL08 & 15 & 2.71 & 18.4 & 1.38 & 0.964 & 15 \\
\hline & YL09 & 17 & & 18.9 & 1.41 & 0.922 & 17 \\
\hline & YL10 & 19 & 2.71 & 18.5 & 1.45 & 0.869 & 15 \\
\hline
\end{tabular}

\subsection{Methods}

Grain size, density, humidity and structure are the important physical properties of soil, which reflect the essential characteristics of soil and are the important factors affecting the collapsibility of loess. Therefore, four parameters, including particle size composition, dry density, initial water content and comprehensive structural potential, were selected to characterize the physical properties of loess, and the correlation between these parameters and the coefficient of collapsibility was 
explored. The test method of each part and the sample preparation process are as follows:

\subsubsection{Influence of initial moisture content on the coefficient of collapsibility}

The moisture content experiment was carried out in three groups, which were named as "JY Group", "LZ Group" and "YL Group" respectively. The moisture content of each sample was changed, and the collapsibility coefficient was measured by the double-line method. In order to ensure the same dry density, JY03, LZ03 and YL07 samples were selected for the test. Each group contained 9 samples, and the water content was controlled at $5 \%, 7 \%, 9 \%, 11 \%, 15 \%, 20 \%, 25 \%$ and $30 \%$. For samples with lower moisture content than natural moisture content, air drying method was adopted to reduce moisture content to the target value. On the contrary, water film transfer method was used. The type of triple consolidation instrument used in the collapse test is GDG-4S, the sample area is $30 \mathrm{~cm}^{2}$, and the height is $2 \mathrm{~cm}$. The loading grades are $12.5 \mathrm{kPa}, 25 \mathrm{kPa}, 50 \mathrm{kPa}, 100 \mathrm{kPa}, 200 \mathrm{kPa}, 300 \mathrm{kPa}$, $400 \mathrm{kPa}$.

\subsubsection{Experimental study on the effect of dry density on collapsibility coefficient}

As the dry density of samples at each sampling point changes with the increase of depth, the moisture content of each sample is controlled to be $7 \%$. The collapsibility coefficient was measured by double - line method.

\subsubsection{Experimental study on the effect of particle size composition on collapsibility coefficient}

In order to determine the particle size composition and collapsibility coefficient of soil samples in each area and analyze their correlation, it is necessary to control the same moisture content and dry density of the samples. Therefore, the collapsibility test results of several groups of samples with similar dry density in 2.2.2 were selected for the analysis in this part, and the particle size composition of each sample was measured by laser particle size analyzer. The sample numbers of each group are as follows: (a) JY01, LZ01, YL06 (b): JY02, LZ05, YL08 (c): JY03, LZ03, YL07 (d): JY06, LZ06, YL09 (e): JY08, LZ09, YL10.

\subsubsection{Experimental study of structural influence on the coefficient of collapsibility}

The comprehensive structural potential is used to characterize the structural strength of loess [16], and its definition is as follows:

$$
m_{p}=\frac{m_{1}}{m_{2}}=\frac{S_{s} / S_{o}}{S_{o} / S_{r}}=\frac{S_{r} \cdot S_{s}}{S_{o}^{2}}
$$

In the formula, $S_{o}, S_{s}$ and $S_{r}$ are respectively the deformation of undisturbed sample, undisturbed saturated sample and remolded sample under a certain pressure.
Therefore, lateral confining compression tests were carried out on the original samples, remolded samples with different water content (water content and dry density were the same as the original samples) and the original saturated samples, and the water content Settings, experimental instruments and loading levels were the same as those in test 2.2.1. In this part, only the confined compression experiments of the remolded samples and the original saturated samples were carried out, and the data of 2.2.1 were used for the confined compression experiments and the collapse coefficient of the original samples. Since the comprehensive structural potential of the sample changes gradually with the increase of pressure, the maximum value of the comprehensive structural potential of the sample is selected for analysis in this paper.

\section{Results and Discussion}

\subsection{Correlation between collapsibility coefficient and initial moisture content}

The initial moisture content of the samples at each sampling point was changed to measure their collapsibility coefficient, and the results were shown in Fig. 1. It can be seen from the figure that under the natural moisture content, the collapsibility coefficient of Jingyang soil sample (JY03) is 0.023 , indicating that it is a slightly collapsible loess. The collapsibility coefficients of Lanzhou and Yili soil samples (LZ03 and YL07) are 0.051 and 0.053 , respectively, indicating that both of them are moderately collapsible loess.

As shown in Figure 1, the collapsibility coefficient of soil samples from Jingyang, Lanzhou and Yili is negatively correlated with the initial water content. When the water content is low, the decrease rate of the collapsibility coefficient is faster. This is because the increase of the initial moisture content of samples reduces the matric suction between the soil particles, the cementing effect of the carbonate cements is weakened, and the compressibility of samples is enhanced. However, for the same kind of soil, the change of water content does not affect the total deformation (including compression deformation and collapse deformation) because of its constant porosity ratio. Therefore, when the initial water content increases, the collapsibility of the sample decreases and the collapsibility decreases. When the initial water content is high, the influence of the change of water content on matric suction and cementation is reduced, so the change of the coefficient of collapsibility is small when the water content is high. In addition, the overall collapsibility coefficient of Jingyang soil sample is slightly lower than that of Lanzhou soil sample, and the collapsibility coefficient of Yili soil sample is the strongest. However, when the initial water content is $30 \%$, the collapsibility coefficient of the three soil samples is basically the same. In addition, on the whole, the collapsibility coefficient of Jingyang soil sample is slightly lower than that of Lanzhou soil sample, and the collapsibility of Yili soil sample is the strongest. However, when the initial moisture content is $30 \%$, the collapsibility coefficients of the three are basically the same. 


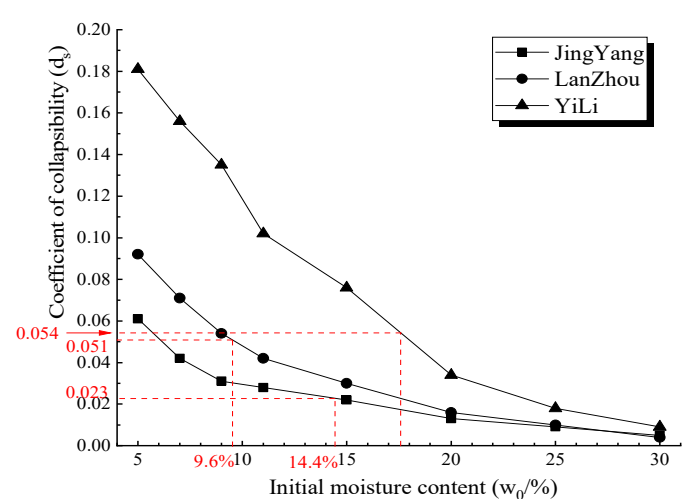

Figure 1. Variation curve of the sample's collapsibility with the initial moisture content at each sampling point

\subsection{Correlation between collapsibility coefficient and dry density}

The dry density of each sample changes gradually with the increase of the buried depth. Therefore, the correlation between the collapsibility coefficient and the dry density is analyzed by measuring the collapsibility coefficient of samples with different buried depths at each sampling point. The results are shown in Figure 2. As can be seen from Fig. 2, the collapsibility coefficient of each sample is in approximately linear negative correlation with the dry density. The analysis shows that the collapsible deformation of loess depends on the size of the collapsible space. When the dry density of soil is small, its structure is looser. In this case, a large number of pores will be filled with soil particles after the structure is destroyed, resulting in large collapsible deformation and strong collapsibility.

By comparing the test results of soil samples from the three regions, it can be seen that the overall collapsibility coefficient of soil samples from Yili is the highest, followed by that from Lanzhou, and the collapsibility of soil samples from Jingyang is slightly lower than that from Lanzhou. The study on the collapsibility of the loess in Yili area shows that the loess in this area was formed in a late age, was less affected by the humid and hot climate, and its natural structure was less damaged, and its structure was dominated by point contact and overhead arrangement, with less cementation. This structural feature of the Yili loess leads to its strong collapsibility [17].

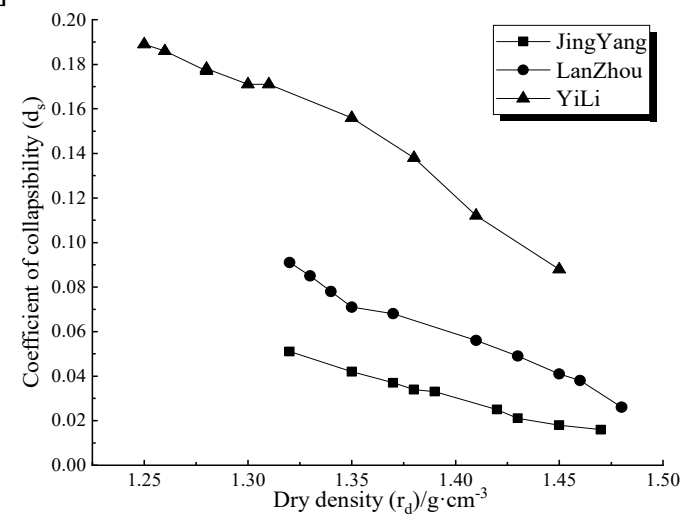

Figure 2. Change curve of the sample's collapsibility coefficient with dry density at each sampling point

\subsection{Correlation between collapsibility coefficient and particle size composition}

There is no obvious uniform correlation between the collapsibility coefficient and the particle content of each sample. According to the analysis of the reasons, the grain size composition of the same loess does not change significantly before and after the collapsibility, but the collapsibility of the original soil samples is greatly different from that of the post-collapsibility soil samples. It can be seen that the particle size component does not have an effect on the collapsibility alone.
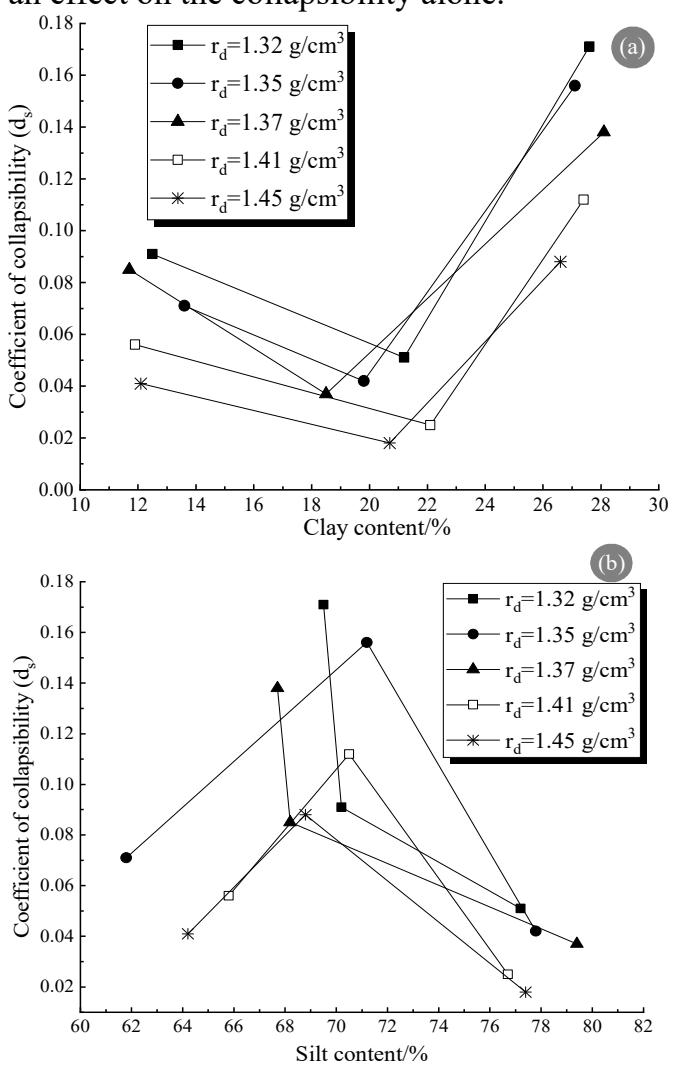

Figure 3. Relation curve between sample collapsibility coefficient and particle size composition at each sampling point:(a) Collapsibility coefficient - clay content;(b)

Collapsibility coefficient - powder content

Fig. 4 shows the microstructure photos of sample JY01 before and after collapsibility. It can be seen from the figure that there are many overhead pores in the original sample, and the skeleton particles are mainly silt particles, while the clay particles are mostly aggregates or attached to the surface of larger particles. After the occurrence of collapsibility, there are still many small clay particles attached to the surface of larger particles. The clay particles existing in the form of adhesion have poor mobility and will not become the main material to fill the pores. The pores are mostly filled by larger clay particles, fine silts or unbroken aggregates. Therefore, when the content of large clay particles (which exist alone or are generated by the fragmentation of aggregates) in the soil is high, and the content of aggregates and fine fine particles with strong cementing effect is high, the filling effect of overhead pores or macropores is strong in the process of collapsibility, and the collapsibility deformation is small. 
From the above analysis, it can be found that the effect of particle size composition on collapsibility is the result of the interaction between particle size composition, clay form and pore characteristics. Therefore, there is no good regularity between the content of clay and silt and the collapsibility.
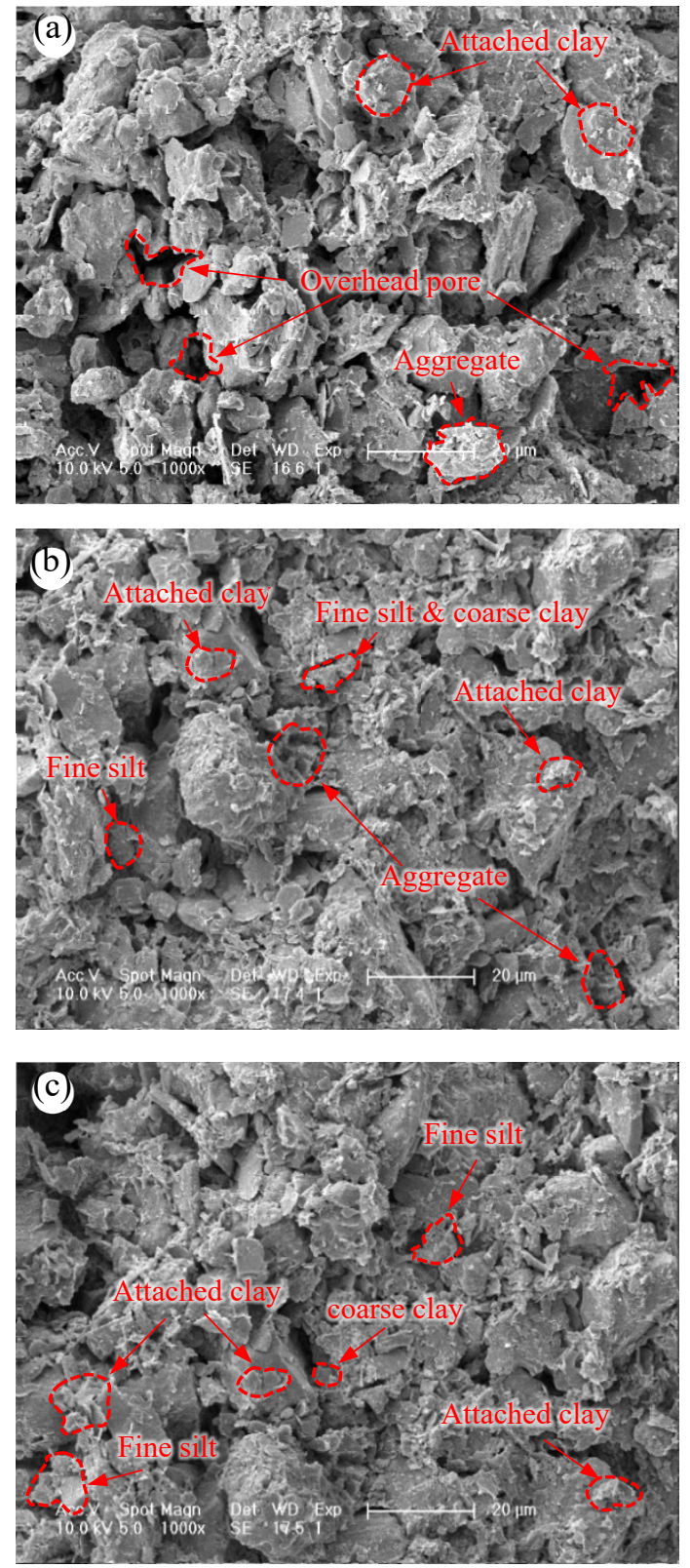

Figure 4. Microstructure photos of JY1 sample before and after wetting:(a) The original sample;(b) Sample after wetting;(c) Sample after wetting

\subsection{Correlation between collapsibility coefficient and composite structural potential}

In different water content states, the relationship curve between the collapsibility coefficient of samples at each sampling point and their comprehensive structural potential is shown in Fig. 5. It can be seen from the figure that the coefficient of collapsibility is in approximately linear positive correlation with the comprehensive structural potential. The samples at each sampling point showed similar rules. When the comprehensive structural potential is small, the collapsibility coefficient of the samples at the three sampling points is basically the same. When the comprehensive structural potential reaches 23.8 , the collapsibility coefficient of the Yili soil sample is obviously higher than that of the Lanzhou soil sample, and with the continuous increase of the comprehensive structural potential, it is expected that the gap will continue to increase. Studies have shown that the comprehensive structural potential is the comprehensive embodiment of soil connection (stability) and arrangement (variability). When the variability is strong, the soil particle bond is destroyed, and the soil structure is in a larger unstable state. Strong structural soil must have strong stability and variability [16]. According to the analysis, when the dry density of soil is small and the content of overhead porosity and macroporosity is high, the soil has strong variability, and the collapsibility deformation is also large. Therefore, the collapsibility coefficient is positively correlated with the comprehensive structural potential.

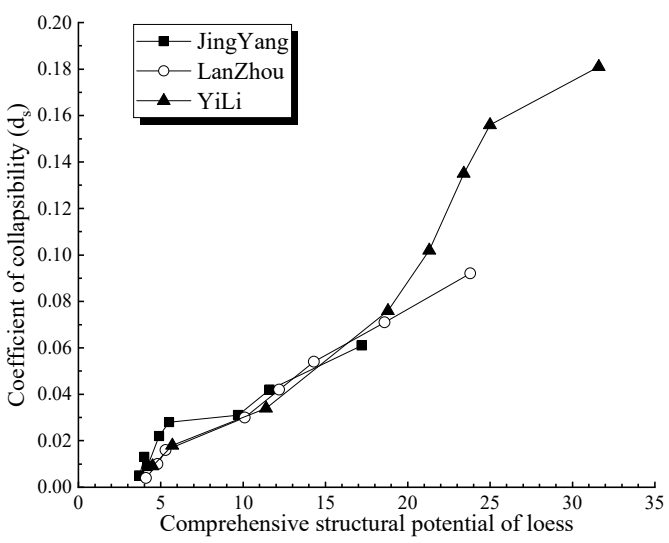

Figure 5. Relation curve of sample collapsibility coefficient and composite structural potential at each sampling point

\section{Conclusions}

Through the collapsibility tests of loess in Jingyang, Lanzhou and Yili under different conditions, the influences of initial moisture content, dry density, particle size composition and structural parameters on loess collapsibility were explored and the reasons were analyzed. The main conclusions are as follows:

1. The collapsibility coefficient of loess in each area is negatively correlated with the initial water content, and the decrease rate of the collapsibility coefficient is greater when the initial water content is lower. The reason is that when the initial water content is high, the compressibility of soil is enhanced, the collapsibility deformation is reduced, and the collapsibility coefficient is also reduced.

2. There is a negative linear correlation between the collapsibility coefficient and dry density. On the whole, the collapsibility of Yili loess is the strongest, while that of Jingyang loess is the weakest. When the dry density of soil is large, the collapsible space decreases and the collapsibility weakens.

3. The collapsibility has a certain correlation with the clay content. When the clay content is about $20 \%$, 
the collapsibility is the weakest. There is no obvious uniform correlation between collapsibility and silt content. It is concluded that the effect of particle size composition on collapsibility is based on the characteristics of pore structure, and is also related to the form of clay particles.

4. The analysis shows that the strong structural soil has high content of overhead pores and macropores, so it has strong variability and strong collapsibility. Therefore, the collapsibility coefficient of loess in each area is approximately linear positive correlation with the comprehensive structural potential.

\section{References}

1. Liu Zudian. Loess mechanics and engineering [M]. Xi'an: Shaanxi Science and Technology Press, 1996.

2. Fang Xiangwei, Shen Chunni, Li Chunhai, et al. Collapsible deformation properties of Q2 loess in Pucheng of Shaanxi province [J]. Rock and Soil Mechanics, 2013, 34(S2):115-120.

3. Wang Jian. Study on the deformation law of undisturbed loess under true triaxial compression and humidification[D]. Xi'an: Xi'an University of Technology, 2020.

4. Fang Jinjin, Feng Yixin, Yu Yongqiang, et al. Humidification and deformation characteristics of undisturbed loess under true triaxial conditions [J]. Rock and Soil Mechanics, 2020, 41(4): 1235-1246.

5. Hou Xiaokun. Research on Deformation Characteristics of Loess Unsaturated Collapsibility[D]. Xi'an: Chang'an University, 2015.

6. Zheng Jianguo, Deng Guohua, Liu Zhenghong, et al. Influence of discontinuous distribution of loess collapsibility on collapse deformation[J]. Chinese Journal of Geotechnical Engineering, 2015, 37(1):165-170.

7. Mi Wenjing, Zhang Aijun, Guo Minxia. Research on the Deformation Characteristics of Yili Loess Collapsible[J]. Jiangxi Journal of Agriculture, 2019, 31(12):49-53.

8. Niu Lisi, Zhang Aijun, Wang Yuguo, et al. Compression and collapsibility characteristics of Yili loess under changes of humidity and density[J]. Journal of Hydroelectric Engineering, 2020.

9. Li Ping, Li Tonglu. The relationship between physical properties of loess and collapsibility and its engineering significance $[\mathrm{J}]$. Journal of Engineering Geology, 2007, 15(4):506-511.

10. Ma Yan, Wang Jiading, Peng Shujun, et al. The relationship between collapsibility of loess and soil properties and its prediction model[J]. Bulletin of Soil and Water Conservation, 2016, 36(1):120-128.

11. Wu Xiaopeng, Zhao Yonghu, Xu Anhua, et al. The relationship between collapsibility of loess and its physical and mechanical indexes and evaluation methods[J]. Journal of Yangtze River Scientific Research Institute, 2018, 35(6):75-80.
12. Li Yongwei, Zhang Peiheng, Li Zhenxiao. Collapsibility of Lishi Loess in Shanxi[J]. Journal of Engineering Geology, 2014, 22(sl):395-399.

13. $\mathrm{Mu}$ Qingyi, Dang Yingjie, Dong Qi, et al. Comparative test study on water holding characteristics and collapsibility of undisturbed and compacted loess[J]. Chinese Journal of Geotechnical Engineering, 2019, 41(8):1496-1504.

14. Cui Jingyu, Zhang Wuyu, Xie Banglong, et al. Collapsibility and microscopic mechanism of undisturbed loess at different depths in Xining area[J]. Chinese Journal of Geotechnical Engineering, 2019, 41(s2):249-252.

15. Fang Xiangwei, Ou Yixi, Shen Chunni, et al. Research on the influencing factors of Q2 loess collapsibility $[\mathrm{J}]$. Journal of Water Resources and Architectural Engineering, 2016, 14(1):49-54.

16. Xie Dingyi, Qi Jilin. A new way to study soil structure and its quantitative parameters[J]. Chinese Journal of Geotechnical Engineering, 1999, 21(6):651-656.

17. Zhang Aijun, Xing Yichuan, $\mathrm{Hu}$ Xinli, et al. Analysis of influencing factors of strong self-weight collapsibility of Ili loess[J]. Chinese Journal of Geotechnical Engineering, 2016, 38(0z2):117-122. 